\title{
Effect of Double Oxide Film Defects on Mechanical Properties of As-Cast C95800 Alloy
}

\author{
Xin-Yi Zhao ${ }^{1,2} \cdot$ Zhi-Liang Ning ${ }^{1,2} \cdot$ Fu-Yang Cao ${ }^{1,2} \cdot$ Shan-Guang Liu ${ }^{1,2} \cdot$ \\ Yong-Jiang Huang ${ }^{1,2} \cdot$ Jing-Shun Liu $^{3} \cdot$ Jian-Fei Sun ${ }^{1,2}$
}

Received: 1 July 2016/Revised: 29 September 2016/Published online: 5 January 2017

(c) The Chinese Society for Metals and Springer-Verlag Berlin Heidelberg 2017

\begin{abstract}
The morphology of double oxide film defects and their influence on the tensile mechanical properties of a commercial $\mathrm{Cu}-\mathrm{Al}$ (C95800) alloy were investigated in this study. Plane castings were produced with two types of pouring systems, and their tensile properties were measured and then analyzed by means of Weibull statistics method. The fracture surfaces of the tensile specimens were examined using scanning electron microscopy equipped with energy-dispersive spectroscopy. A large amount of double oxide film defects were observed on the tensile fractured specimens of the topfilled plane castings, and their chemical composition is identified to be $\mathrm{Al}_{2} \mathrm{O}_{3}$. Weibull statistics analyses showed that the double oxide film defects significantly reduce mechanical properties of the castings investigated. Furthermore, the ultimate tensile strength is more obviously deteriorated by double oxide film defects than elongation.
\end{abstract}

KEY WORDS: Double oxide film defects; C95800 alloy; Running system; Weibull statistics analysis

\section{Introduction}

Double oxide films are generated as a result of the flow surface breaking waves during filling process with sufficiently high velocity (namely above the critical velocity). The corresponding formation mechanisms have been well documented in Refs. [1-3]. Due to the serious surface turbulence, oxidized surfaces of the melt will fold over and immerse into the bulk liquid with a trapped layer of local atmosphere. Therefore, the morphology of the double

Available online at http://link.springer.com/journal/40195

Jian-Fei Sun

jfsun_hit@263.net

1 School of Materials Science and Engineering, Harbin Institute of Technology, Harbin 150001, China

2 National Key Laboratory for Precision Hot Processing of Metals, Harbin Institute of Technology, Harbin 150001, China

3 School of Materials Science and Engineering, Inner Mongolia University of Technology, Hohhot 010051, China oxide film defects is similar to sandwich [4]. As the surface oxide film of $\mathrm{Al}$ alloys can transform from amorphous alumina to crystalline $\gamma-\mathrm{Al}_{2} \mathrm{O}_{3}$, then to $\alpha-\mathrm{Al}_{2} \mathrm{O}_{3}$, at last to magnesium aluminate spinel $\mathrm{MgAl}_{2} \mathrm{O}_{4}$ along with oxidation time, the oxide film defects in $\mathrm{Al}$ alloys are normally unbounded alumina or spinel [5]. Many investigations on the failure of the as-cast $\mathrm{Al}$ alloys have identified that double oxide films caused the defects commonly occurred in castings on account of their crack-like feature, such as hydrogen-induced porosities, cracks of second phases, pores and hot cracks, which obviously deteriorated the ultimate tensile strength, elongation and fatigue property of the alloys [6-10].

Nyahumwa et al. [11] revealed the effect of surface turbulence on the fatigue strength of as-cast $\mathrm{Al}-7 \mathrm{Si}-\mathrm{Mg}$ alloys and found that the fatigue cracks initiated at the double oxide film defects. Based on the Weibull modulus results of the UTS and elongation of castings fabricated under different filling conditions varying from quiescent to strong turbulence, Mi et al. [12] found that the amount of entrained oxide films was closely related to tilt pouring parameters and these films were responsible for the 
reduced reliability of castings. Dai et al. [13] and Yang et al. [14] demonstrated that an improper design of the filling system would result in undesired higher metal flow velocity and surface turbulence. Accordingly, the surface oxide films on the liquid advancing front were folded-over and entrapped into the bulk liquid metal. In order to acquire a proper filling velocity (lower than the critical velocity, $0.5 \mathrm{~m} / \mathrm{s}$ for Al alloy) and reduce the tendency of the formation of double oxide film defects, considerable efforts focusing on the design of running system have been conducted [15-18].

Besides Al-based alloys, other alloys covered with surface oxide films are also expected to form double oxide film defects. Griffiths and Lai [19] found that the oxide film defects were comprised of the folded over and symmetrical $\mathrm{MgO}$ films in a commercial purity $\mathrm{Mg}$ alloy, similar to the defects found in Al-based alloys in form and structure. Campbell and Tiryakioğlu [20] found the double oxide film defects in Ni-based casting alloy, indicating that these defects were inevitable even melting and casting in vacuum environment. They believed that counter gravity filling was an important method to produce almost defect-free castings. However, the double oxide film defects were also found in the low pressure cast A356 Al alloys and the formation mechanism was elaborated in our recent work [21].

Up to now, studies concerning the formation of the double oxide film defects and their influences on the mechanical properties are mainly focused on the light alloys, i.e., $\mathrm{Al}$ alloys and $\mathrm{Mg}$ alloys. Generally, bronze alloy melt is oxidizable, which is expected to form the double oxide film defects in the castings. Due to the great density difference between oxide films and the melt, the movement of double oxide film defects is more easily affected by filling flow, especially for $\mathrm{Cu}-\mathrm{Al}$ alloy. Therefore, comparing with the case of light-weight alloys, the filling turbulence of $\mathrm{Cu}-\mathrm{Al}$ melt caused by poor filling system design and complicated cavity structure may largely decrease the mechanical properties of castings. However, very limited information on the formation of double oxide films and their effects on the mechanical properties of $\mathrm{Cu}-\mathrm{Al}$ alloys have been reported so far. In this paper, the double oxide film defects in the castings produced by the different pouring systems were observed and their effects on the mechanical properties of C95800 alloy were further investigated.

\section{Experimental}

Two filling manners were designed to represent the two different practices in running system design, poorly designed and well-designed, as shown in Fig. 1. The dimensions of the cast plate are $50 \mathrm{~mm}$ in height, $80 \mathrm{~mm}$ in breadth, and $15 \mathrm{~mm}$ in width. In order to produce surface turbulence and considerable oxide films entrainment, the first series of castings were directly filled from the top into the vertically oriented plates without filter, as shown in Fig. 1a. The second series of plate castings were poured through the bottom-filled running system, along with a tapered sprue, a 15 ppi ceramic foam filter with a thickness of $20 \mathrm{~mm}$ and a thinner runner, as shown in Fig. $1 \mathrm{~b}$ for the purpose to obtain less double oxide film defects. Due to the great density difference between oxide film and liquid metal, and the small size of castings, the double oxide film defects may easily move into the feeder driven by flow, decreasing the content of oxide film in castings. Therefore the castings were designed without feeder.

The height $(H)$ of the horizontal runner (in Fig. 1b) below the filter for preventing the turbulence of flow surfaces and formation of double oxide film defects was calculated by Eq. (1) according to Ref. [22]: (a)

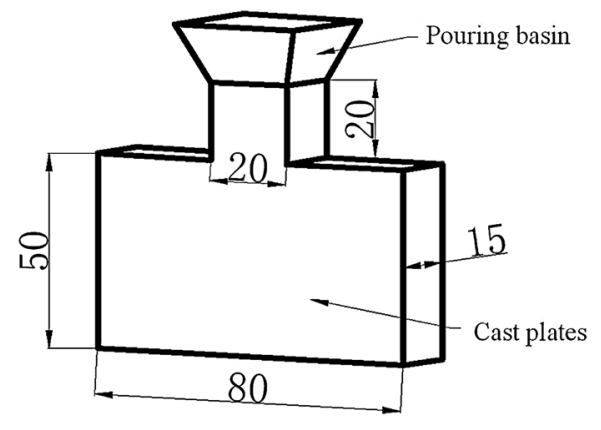

(b)

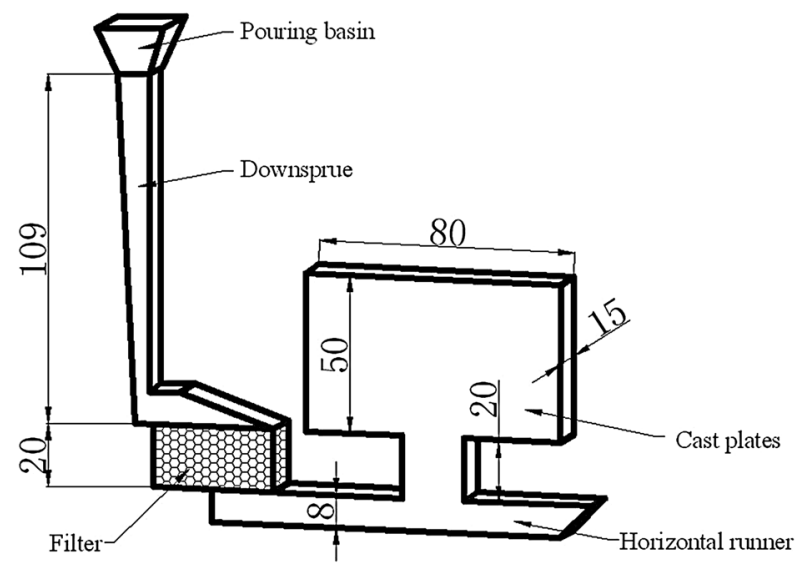

Fig. 1 Schematic illustrations of the two-type filling manners for the plate casting with the same dimensions $(80 \mathrm{~mm} \times 50 \mathrm{~mm} \times 15 \mathrm{~mm})$ : a top-filling running system and filter, $\mathbf{b}$ the bottom-filled running system with a 15 ppi ceramic filter (unit: $\mathrm{mm}$ ) 
$H \leq \frac{v^{2}}{2 g}$.

where $v$ is the critical velocity of the liquid metal flow, which is a function of surface tension and density of the liquid metal. It was estimated as about $0.4 \mathrm{~m} / \mathrm{s}$ for $\mathrm{Cu}$ alloy suggested by Campbell [23]. The maximum $H$ was determined to be $8.16 \mathrm{~mm}$, according to Eq. (1). Therefore, $H$ was designed as $8 \mathrm{~mm}$ in this study, as shown in Fig. $1 b$.

A commercial C95800 alloy was used to fabricate the castings, and its chemical compositions are given in Table 1. In order to avoid the oxidation of melt, the alloy was melted in an induction furnace covered with charcoal, and less stirring was operated during the melting process. After melting, the liquid metal was held at $1300{ }^{\circ} \mathrm{C}$ for $10 \mathrm{~min}$ for chemical compositions uniformity and the immersed oxidations floating out. Then, the plates were cast at $1150{ }^{\circ} \mathrm{C}$ immediately after slagging off surface oxidation. The molds were manufactured by sodium silicate-bonded sand and hardened through blowing $\mathrm{CO}_{2}$. The filling processes of the two-type molds were simulated by commercial Flow-3D software, and a turbulence model "two-equation $(k-e)$ model" was employed in order to analyze the turbulence filling.

Two castings were fabricated for each pouring manner, and 18 tensile specimens with a gauge section of $5.0 \mathrm{~mm} \times 1.5 \mathrm{~mm}$ were cut from each of the as-cast castings. In order to eliminate the effect of surface and edge, all specimens were taken from the center of castings, as shown in Fig. 2. The tensile tests were conducted on an Instron-5569 electronic universal materials testing machine

Table 1 Compositions (wt\%) of the commercial C95800 alloy

\begin{tabular}{llllllll}
\hline $\mathrm{Al}$ & $\mathrm{Fe}$ & $\mathrm{Ni}$ & $\mathrm{Mn}$ & $\mathrm{Pb}$ & $\mathrm{Si}$ & $\mathrm{C}$ & $\mathrm{Cu}$ \\
\hline 8.76 & 1.62 & 4.31 & 1.98 & 0.01 & 0.08 & 0.09 & Bal. \\
\hline
\end{tabular}

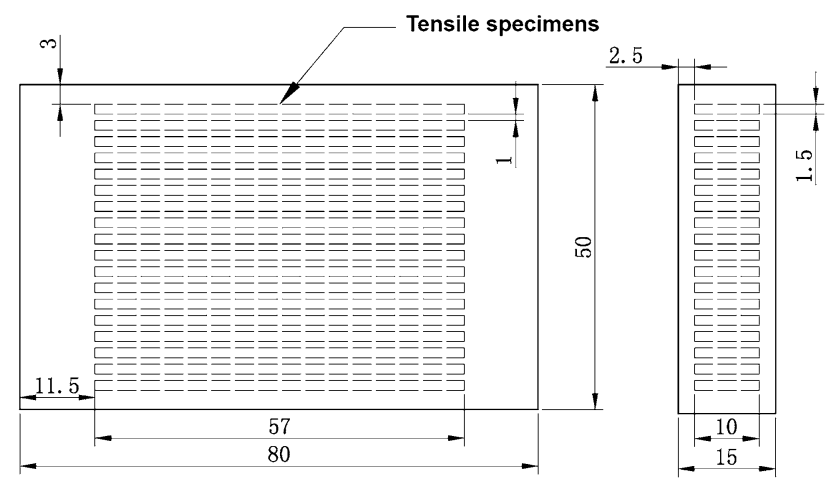

Fig. 2 Sketch of the casting and the tensile test specimens with an initial strain rate of $8.0 \times 10^{-4} / \mathrm{s}$. The fractured surfaces of the specimens were examined using a Helios Nanolab600i scanning electron microscope (SEM) and the compositions of oxide films were determined by energydispersive spectrometer (EDS).

Weibull analysis of the mechanical properties for castings was widely adopted to study the effect of double oxide film defects on fracture reliability. The cumulative probability function of the Weibull distribution is expressed as follow [24]:

$P=1-\exp \left[-\left(\frac{x-x_{T}}{x_{0}}\right)^{m}\right]$

where $P$ is the possibility of failure at a given value $x$ (generally, it represents either UTS or elongation), the threshold value $x_{T}$ is the lowest value expected to failure, and the terms $x_{0}$ and $m$ are used to characterize the distribution of measured properties, denoted as scale parameter and Weibull modulus, respectively. According to the method of two-parameter Weibull distribution analysis, setting $x_{T}=0$ (assuming the lowest UTS or elongation equals to zero), and Eq. (2) can be described as follow:

$P=1-\exp \left[-\left(\frac{x}{x_{0}}\right)^{m}\right]$

Equation (3) can be re-written as below:

$\ln [-\ln (1-P)]=m \ln (x)-m \ln \left(x_{0}\right)$.

\section{Results and Discussion}

\subsection{Simulation of Filling Process}

Figure 3 shows the simulation results of the filling process for the top-filled manner. Liquid metal poured from gating fills the mold fluently before colliding the bottom of the mold. This filling process can be regarded as free-fall under gravity. The liquid metal flow velocity reaches $1.4 \mathrm{~m} / \mathrm{s}$ when encounters the bottom of the mold, even giving that the initial filling velocity is 0 . It is much larger than the critical filling velocity of $0.4 \mathrm{~m} / \mathrm{s}$ for Cu alloy. Even though colliding the bottom of the mold would bring large energy loss, the liquid metal still keeps enough velocity to collide with bilateral vertical walls and forms jet upward, as shown in Fig. 3a. Figure $3 b$ indicates that the dropping back fluid induces a large amount of oxide film immersion and gas entrainment, resulting in appropriate formation of two symmetrical vortexes. The two vortexes keep generating double oxide film defects until the end of filling (Fig. 3c). Due to the great density difference between liquid metal and oxide film, the entrained double oxide films were 

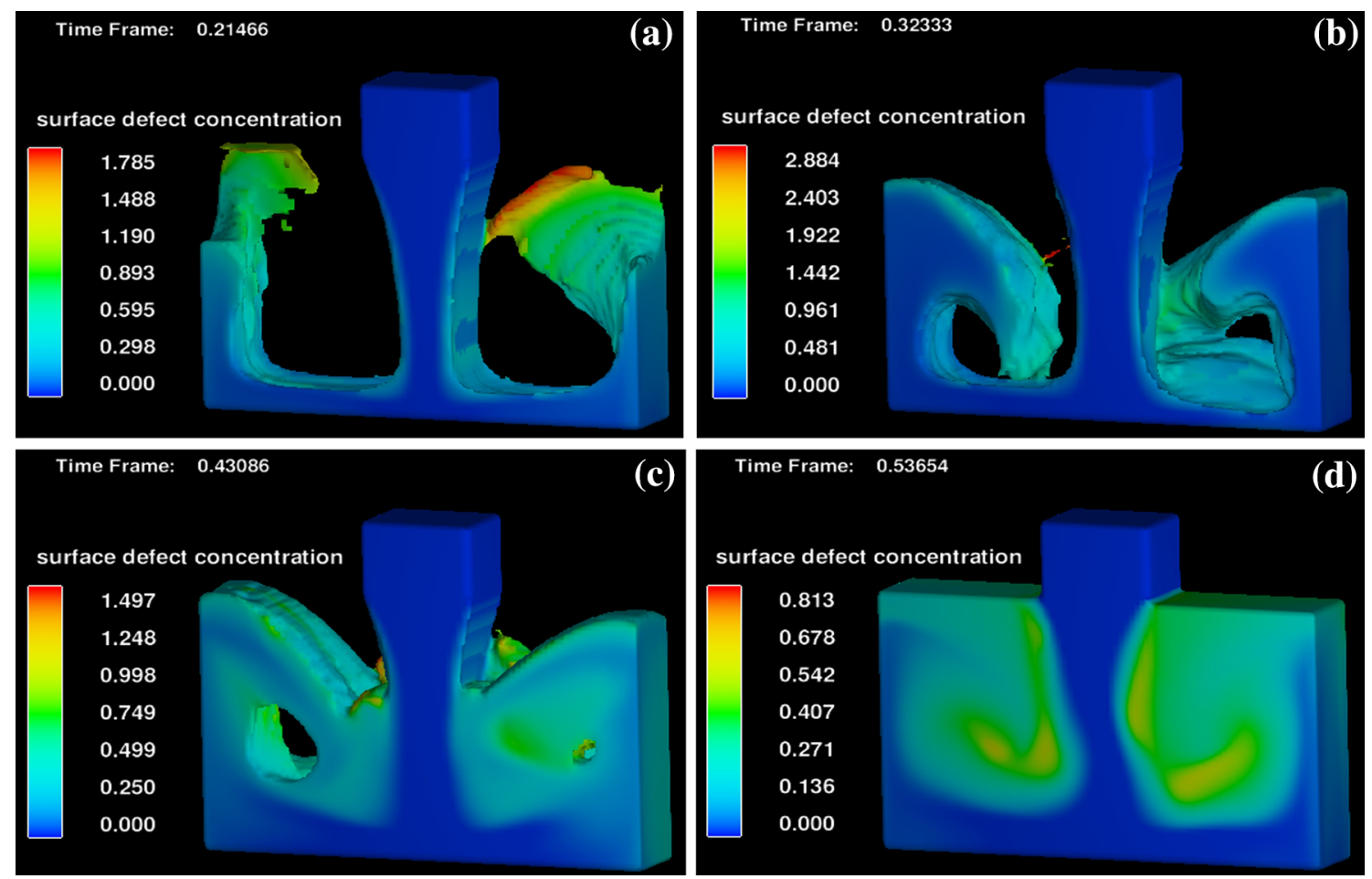

Fig. 3 Simulation results of turbulence filling process of top-filled mold: a the liquid metal collided with bilateral vertical walls and formed jet upward, $\mathbf{b}$ jet dropping back and causing oxide film immersion and gas entrainment, $\mathbf{c}$ formation of two vortexes, $\mathbf{d}$ distribution of oxide defects after filling

distributed in terms of fluid field and mainly located at the both sides of gating, as shown in Fig. 3d.

The simulated mold filling processes for the bottomfilled manner are given in Fig. 4, showing that the melt flows slowly and smoothly without surface turbulence formation, as shown in Fig. 4a. This can be contributed to the combination of well-designed running system and essential performance of filters. It has been previously reported that the gating velocity can be substantially slowed down by the large flow resistance of filter which changed the turbulent flow into laminar flow [25]. As shown in Fig. 4b, a small hump appears on the fluid front surface during filling, which is probably induced by the jet flow of gating. However, the corresponding surface wave is not strong enough to involve surface oxide into the bulk of metal. Consequently, most oxide defects present at the top corners of the castings as expected due to the upward driven force of liquid flow stream and buoyancy.

\subsection{Fracture Surface Observations}

Tensile tests for the castings produced by both pouring manners were conducted, and SEM observations were employed to examine the fracture surfaces. A quantity of wrinkles is clearly detected from the top-filled specimens, as shown in Fig. 5a. EDS analyses show a large amount of oxygen $(\sim 33.48$ at. $\%)$ on these wrinkles, and their compositions are presented in Fig. 5b. Moreover, the atomic ratio of oxygen to aluminum is approximately $3 / 2$, demonstrating that these wrinkles on the fractured surfaces are $\mathrm{Al}_{2} \mathrm{O}_{3}$. This verifies that a large amount of oxide films are induced into the castings during the filling, consistent with the simulated results in Fig. 3a.

The symmetrical structure of oxide film defects was clearly observed in Fig. $6 a, b$, confirming that the defects were folded-over oxide films, and the two layers of films were unwetted. Figure $6 \mathrm{c}, \mathrm{d}$ is the high-magnification images of the oxide films shown in Fig. 6a. The folded and wrinkled appearance of oxide film are observed, which is exactly similar to the double oxide film defects in Mg alloy as depicted in Ref. [19].

For the specimens obtained from bottom-filled castings, the fractured surfaces show small dimples and faceted planes as shown in Fig. 7, much different from those of top-filled ones. However, double oxide film defects were detected in a few bottom-filled specimens, which may be introduced during filling the running system. Assuming that the filling velocity below the filter was zero, increasing the height of runner after filter in running system beyond the critical height of $12 \mathrm{~mm}$ will 

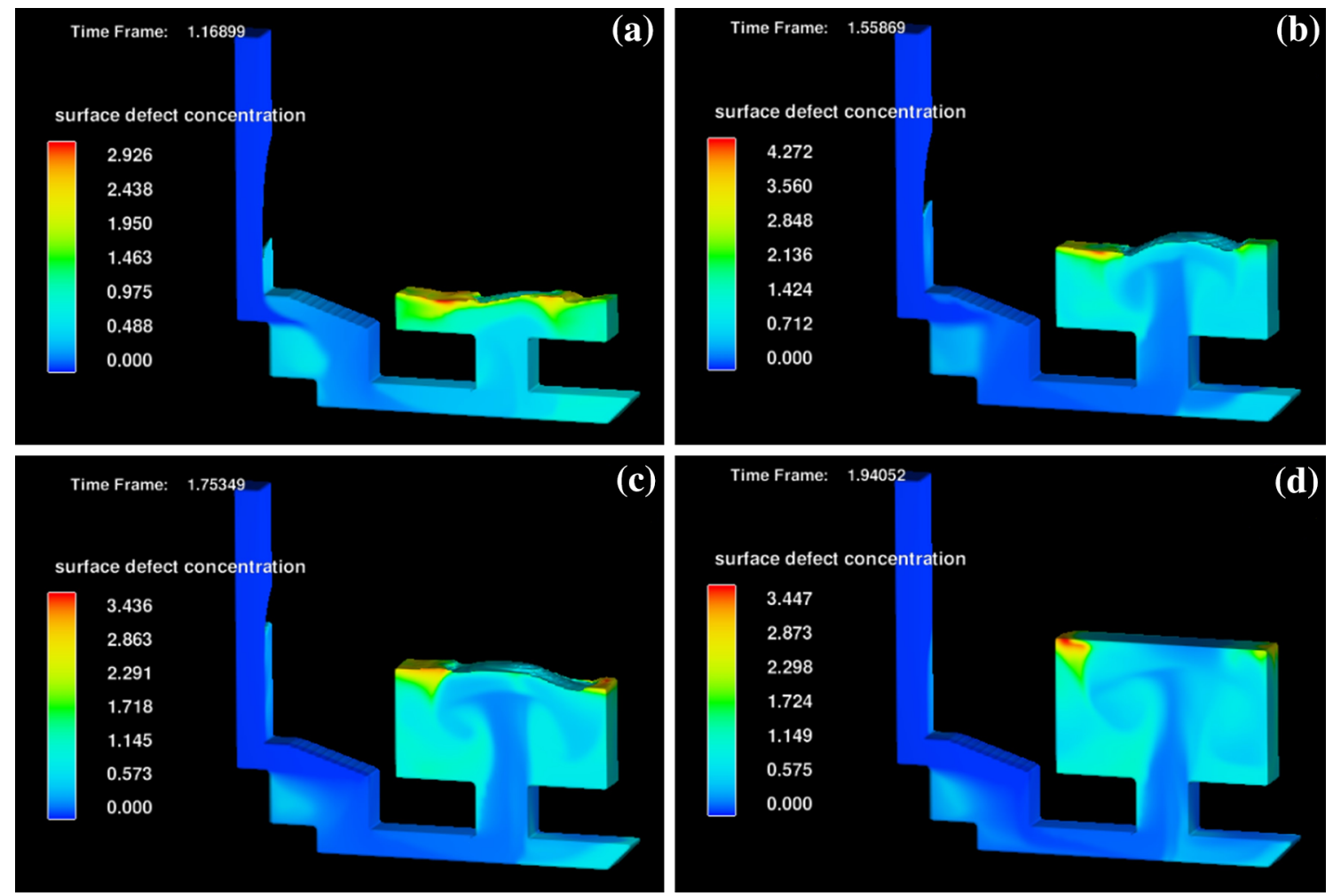

Fig. 4 Simulation results of filling processes for bottom-filled mold $\mathbf{a}-\mathbf{c}$ the smooth filling processes, $\mathbf{d}$ distribution of oxide defects after filling
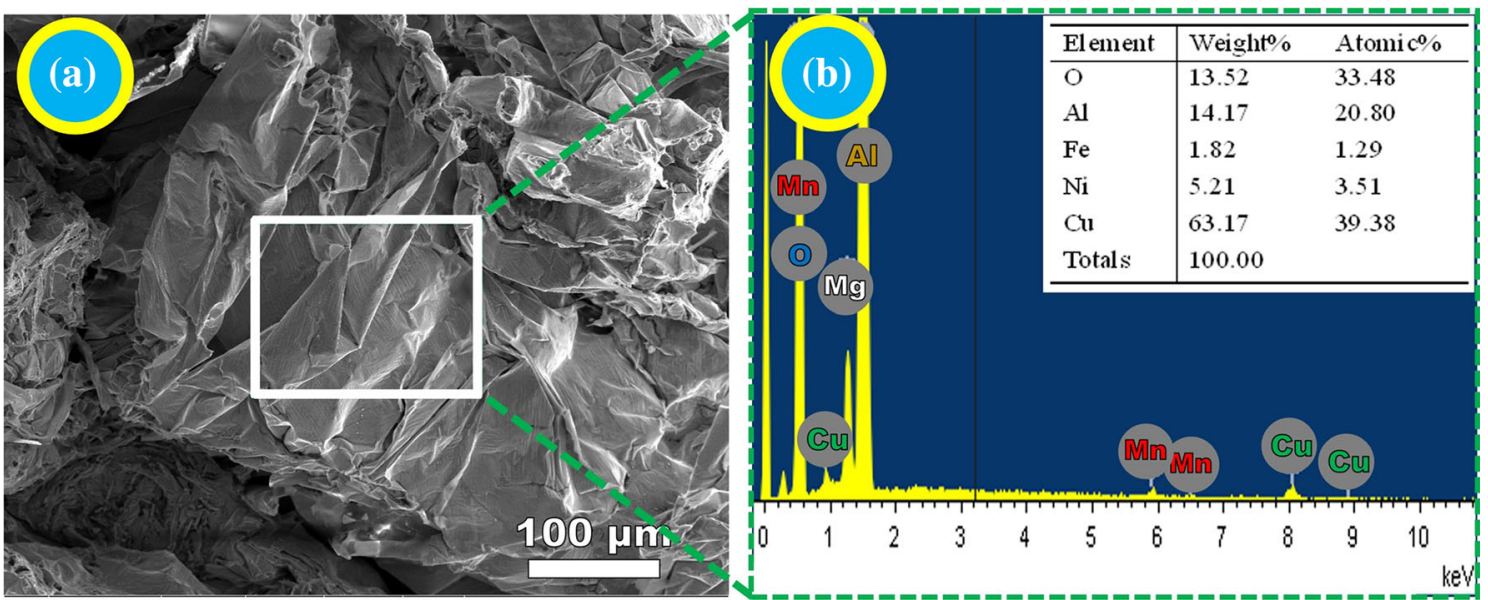

Fig. 5 a SEM image of oxide films on the fracture surface of test specimen taken from a top-filled cast plate, b compositions of the marked area in a analyzed by EDS

degrade the average mechanical properties of $\mathrm{Al}$ alloys castings [22]. However, the assumption underestimated the filling velocity below filter. Campbell suggested that the flow velocity decreased $(75-80) \%$ after passing the 20-ppi filter [26]. Thus, after passing the filter, the real filling velocity cannot be slowed down to zero as assumed. The filling velocity in horizontal runner would be slightly faster than the critical velocity of $0.4 \mathrm{~m} / \mathrm{s}$ for $\mathrm{Cu}$ alloy even the runner height below filter is set as $8 \mathrm{~mm}$ in this work, leading to the formation of a small amount of double oxide films for the bottom-filled castings, as observed on the fracture surfaces by SEM. 

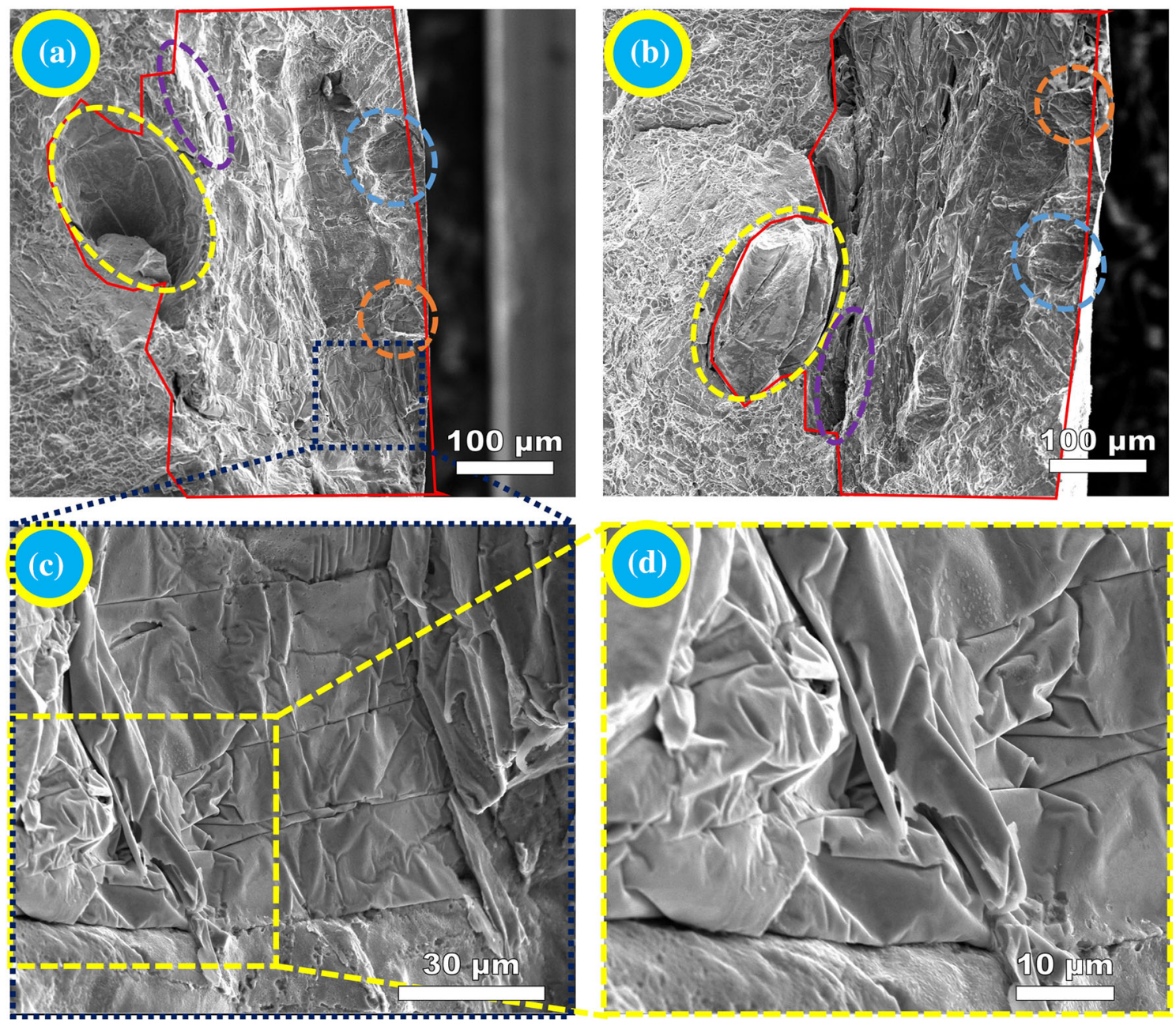

Fig. 6 SEM images of oxide films on two sides $\mathbf{a}$ and $\mathbf{b}$ of the fracture surface of a tensile specimen obtained from a top-filled cast plate, $\mathbf{c}$ higher magnification image of the oxide films in $\mathbf{a}, \mathbf{d}$ the enlarged image of the oxide films shown in $\mathbf{c}$

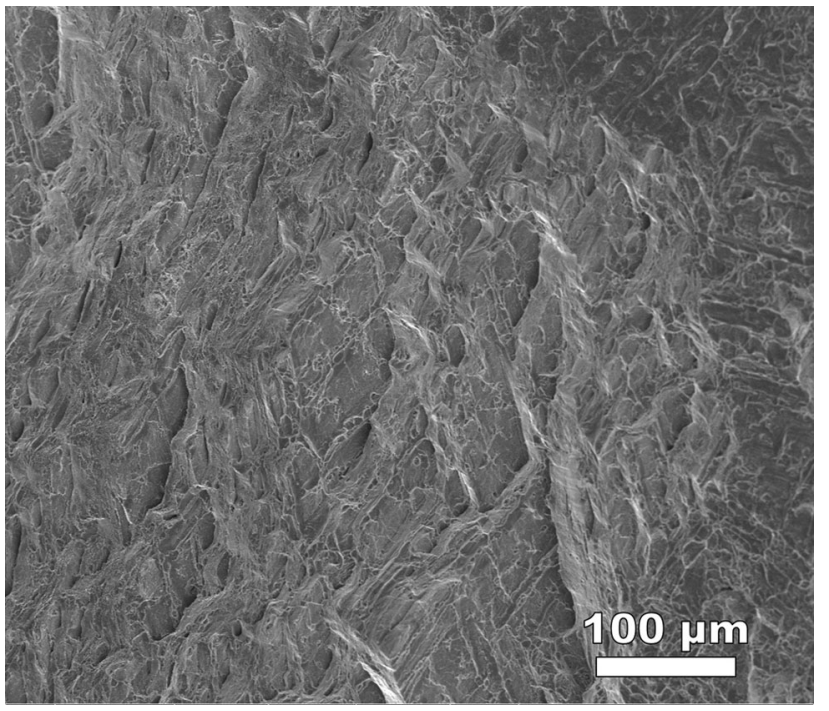

Fig. 7 SEM image of the fractured surface of a tensile specimen taken from a bottom-filled cast plate

\subsection{Mechanical Properties}

The simulation results qualitatively reflect the distribution of double oxide film defects in castings after filling. Based on the Stokes' Law, the limitation floating velocity of oxidation in $\mathrm{Cu}-\mathrm{Al}$ melt can be described as Eq. (5) [27],

$v=\frac{1}{18}\left(\rho_{k}-\rho_{f}\right) \frac{g d^{2}}{\mu}$.

where $\rho_{k}$ and $\rho_{f}$ are densities of oxidation and melt, respectively. $g$ is gravity acceleration, $d$ is the diameter of oxidation, and $\mu$ is the dynamic viscosity of $\mathrm{Cu}-\mathrm{Al}$ melt.

According to the observation of double oxide film defects on fracture surface, the diameter of oxide film was about $\sim 200 \mu \mathrm{m}$. So the floating velocity of oxide film defects was estimated to $9 \times 10^{-8} \mathrm{~m} / \mathrm{s}$. Therefore, even though the density difference between melt and oxidation is relatively large, buoyancy hardly affect the movement of double oxide film defects in the castings. Obviously, flow 
is the main factor of the movement of oxide films. As a result of the vortex generated during filling, oxide defects concentrated in the center of top-filled castings. For bottom-filled castings, oxide defects moved to top surface driven by upward flow. According to the tensile specimens taken method (Fig. 2), it can be inferred that the specimens taken from bottom-filled castings have lower double oxide film defects content than the top-filled. Moreover, the difference of fracture surfaces morphology between top- and bottom-filled casting tensile specimens also confirmed the above speculation. Therefore, the mechanical properties difference between the two kinds of tensile specimens was mainly attributed to the effects of double oxide film defects.

Tensile properties obtained from the top-filled and bottom-filled plates are summarized in Table 2 . The average UTS of top-filled specimens is $\sim 560.9 \mathrm{MPa}$, lower than $612.5 \mathrm{MPa}$ of bottom-filled specimens. As determined statistically in Fig. 8, 40\% specimens from the top-filled plates show the UTS values ranging from 500 to $550 \mathrm{MPa}$. Whereas UTS values of most bottom-filled specimens were higher than $600 \mathrm{MPa}$. The results reveal that the bottomfilled castings exhibit excellent uniformity and higher UTS. However, the average elongation values for top- and bottom-filled castings are $22.8 \%$ and $24.4 \%$, respectively, as

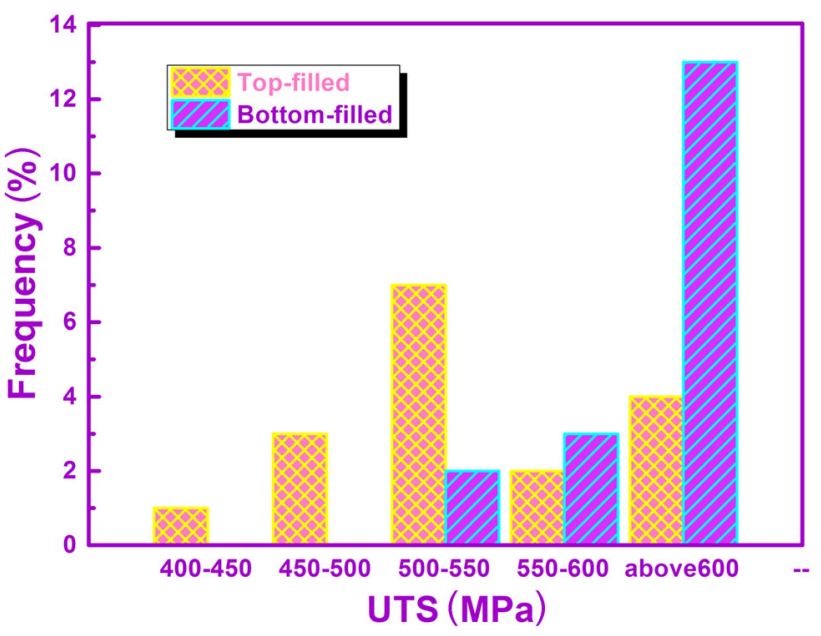

Fig. 8 Distribution of UTS from top-filled and bottom-filled C95800 alloy castings

shown in Table 2. This indicates that the oxide film defect has little effect on elongation.

According to Table 2, the two-parameter and three-parameter Weibull plots for UTS have been obtained and shown in Fig. 9. Two-parameter Weibull modulus $(m)$ of UTS for top-filled castings is $\sim 10.3$, less than that of the bottom-filled plate $(\sim 21.8)$. The scale parameter $\left(\sigma_{0}\right)$ for

Table 2 Tensile properties of castings fabricated by top-filled and bottom-filled pouring systems

\begin{tabular}{|c|c|c|c|c|}
\hline \multirow[t]{2}{*}{ Test number } & \multicolumn{2}{|l|}{ Top-filled } & \multicolumn{2}{|l|}{ Bottom-filled } \\
\hline & UTS (MPa) & Elongation (Pct) & UTS (MPa) & Elongation (Pct) \\
\hline 1 & 525.9 & 23.2 & 545.8 & 21.2 \\
\hline 2 & 521.3 & 27.5 & 575.8 & 24.2 \\
\hline 3 & 509.4 & 25.6 & 633.4 & 21.7 \\
\hline 4 & 513.5 & 24.8 & 601.5 & 19.7 \\
\hline 5 & 461.8 & 20.0 & 629.3 & 27.6 \\
\hline 6 & 515.0 & 26.2 & 618.8 & 26.5 \\
\hline 7 & 435.6 & 18.7 & 643.3 & 29.2 \\
\hline 8 & 480.0 & 17.5 & 597.8 & 23.7 \\
\hline 9 & 495.7 & 16.8 & 545.7 & 27.5 \\
\hline 10 & 528.0 & 18.7 & 621.7 & 22.3 \\
\hline 11 & 540.4 & 14.4 & 598.5 & 21.7 \\
\hline 12 & 560.9 & 20.2 & 636.8 & 21.1 \\
\hline 13 & 572.4 & 21.7 & 613.6 & 24.8 \\
\hline 14 & 609.3 & 27.9 & 657.3 & 28.4 \\
\hline 15 & 616.7 & 27.3 & 626.3 & 20.8 \\
\hline 16 & 630.5 & 29.2 & 625.3 & 25.4 \\
\hline 17 & 630.8 & 28.2 & 639.2 & 25.5 \\
\hline 18 & & & 615.3 & 28.4 \\
\hline Average & 538.1 & 22.8 & 612.5 & 24.4 \\
\hline
\end{tabular}




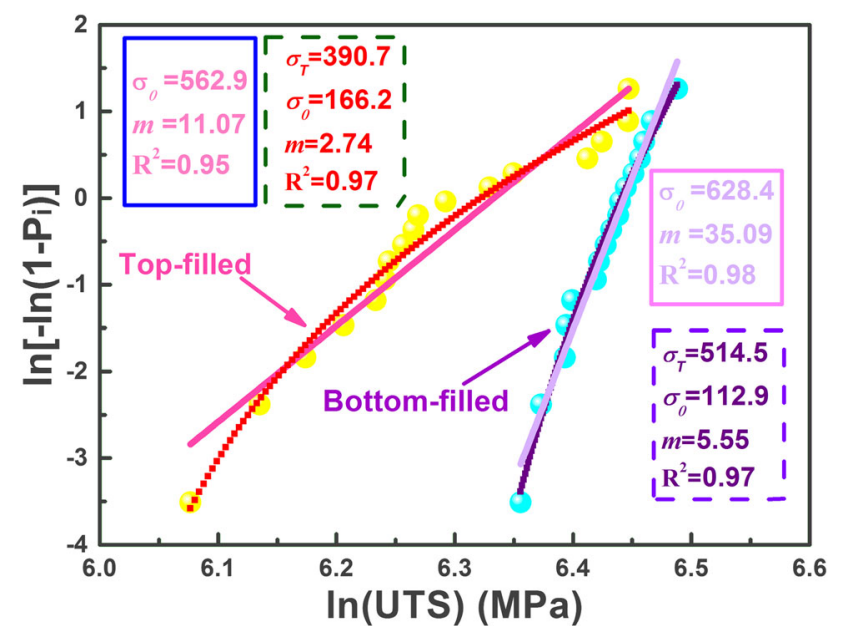

Fig. 9 Weibull plotting of UTS results from top-filled and bottomfilled C95800 alloy castings

top-filled plate is $\sim 562.9 \mathrm{MPa}, \sim 10 \%$ lower than that of the bottom-filled case $(\sim 628.4 \mathrm{MPa})$. The fracture threshold value $\left(\sigma_{T}\right)$ obtained from three-parameter Weibull plot for top-filled specimens is $\sim 390.7 \mathrm{MPa}$, much lower than that of the bottom-filled specimens ( $\sim 514.5 \mathrm{MPa})$. Obviously, the tensile properties of topfilled castings appear more disperse. It can be deduced that the bottom-filled castings exhibit better reliability of the mechanical properties.

Based on the above analysis, the double oxide film defects significantly reduced the UTS and reliability of $\mathrm{Cu}-\mathrm{Al}$ alloy. They can act as natural initiation cracks in the castings account to their doubled-over structure. The running system with bottom-filled mold and filter reduced the entrainment of double oxide film effectively, which results in higher $m$ value in the UTS in compared with that of the top-filled. These results are similar to the previous researches for light alloys [12, 19, 21]. Correspondingly, the double oxide films, resulted from poor design of running system, can exert a crucial role on the mechanical properties.

Moreover, both two-parameter and three-parameter Weibull plots were carried out on elongation for both topand bottom-filled specimens, as shown in Fig. 10. Twoparameter Weibull module $(m)$ for elongation shows the similar trend as UTS, for the value of the bottom-filled alloy is 8.9 , and is slightly higher than $\sim 5.3$ of the topfilled alloy. Meanwhile, the scale parameters for elongation $\left(\varepsilon_{0}\right)$ of the two kinds of castings are very close, which are 24.8 and 25.8 , respectively. It seems that the elongation show less sensitive to double oxide film defects than the UTS. Contrarily, the effect of double oxide film defects in $\mathrm{Mg}$ casting reported by Griffiths indicated that the scale parameter for elongation $\left(\varepsilon_{0}\right)$ was more sensitive to the

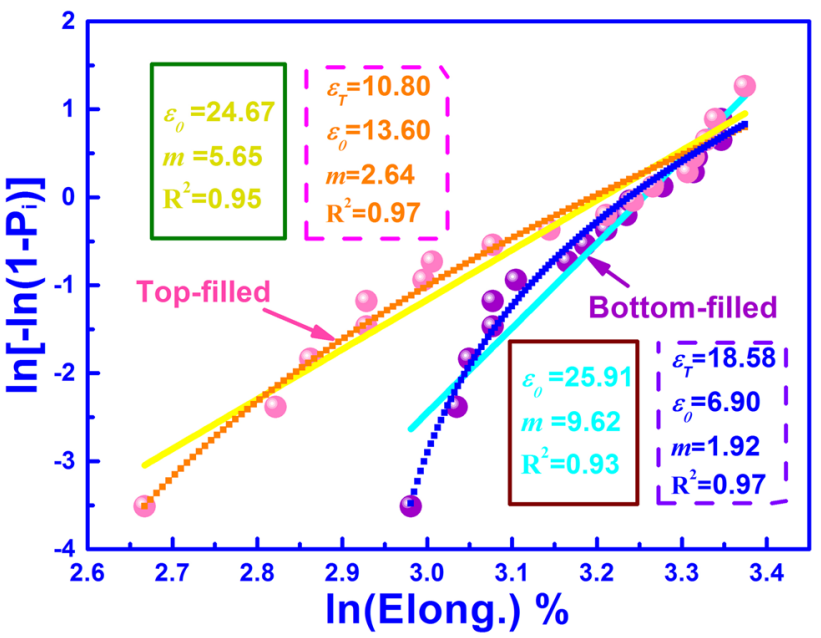

Fig. 10 Weibull plot of elongation results for both top-filled and bottom-filled C95800 alloy castings

defects than the UTS [19]. The insensitive effect of double oxide film defects on elongation of $\mathrm{Cu}$ alloys may be considered as a result of better ductility for $\mathrm{Cu}$ matrix. Even under the tensile condition beyond yield strength, the $\mathrm{Cu}$ alloys can still maintain continuously plastic deformation without failure. The detailed mechanism remains unclear and needs further study.

\section{Conclusions}

The double oxide film defects and their effects on mechanical properties for a C95800 alloy were systematically investigated using two different pouring systems, and the corresponding results are summarized as follows:

1. Numerical simulation and the SEM observation of fracture surfaces indicated that the formation of double oxide film defects in C95800 was significantly affected by pouring manners and filling system design.

2. The double oxide film defects were observed as wrinkle structures appearing on the fracture surfaces of tensile specimens for top-filled casting and identified as $\mathrm{Al}_{2} \mathrm{O}_{3}$ by EDS.

3. Mechanical properties of the castings prepared with both top- and bottom-filled running systems were compared. Double oxide film defects in top-filled castings reduced the UTS and reliability compared with bottom-filled castings. The elongation shows the less sensitive to double oxide film defects than the UTS for this alloy investigated.

Acknowledgements Many thanks are to Dr. H.X. Shen and Dr. Y.D. Jia from Harbin Institute of Technology for useful discussion. 


\section{References}

[1] B. Nayebi, M. Divandari, Int. J. Cast Met. Res. 25(5), 270 (2012)

[2] F. Bahreinian, S.M.A. Boutorabi, J. Campbell, Int. J. Cast Met. Res. 19(1), 45 (2006)

[3] M. Divandari, J. Campbell, Int. J. Cast Met. Res. 17(3), 182 (2004)

[4] J. Campbell, Castings (Butterworth-Heinemann, Oxford, 2003), p. 17

[5] R. Raiszadeh, W.D. Griffiths, Metall. Mater. Trans. 37(6), 865 (2006)

[6] J. Campbell, Metall. Mater. Trans. B 37(6), 857 (2006)

[7] R. Gopalan, N.K. Prabhu, Mater. Sci. Technol. 27(12), 1757 (2011)

[8] R. Raiszadeh, W.D. Griffiths, Metall. Mater. Trans. B 39(2), 298 (2008)

[9] P. Davami, S.K. Kim, N. Varahram, Mater. Sci. Eng. A 552, 36 (2012)

[10] M.A. El-Sayed, W.D. Griffiths, Int. J. Cast Met. Res. 27(5), 282 (2014)

[11] C. Nyahumwa, N.R. Green, J. Campbell, AFS Trans. 106, 215 (1998)

[12] J. Mi, R.A. Harding, J. Campbell, Metall. Mater. Trans. A 35(9), 2893 (2004)
[13] X. Dai, X. Yang, J. Campbell, J. Wood, Mater. Sci. Eng. A 354(1), 315 (2003)

[14] X. Yang, M. Jolly, J. Campbell, Alum. Trans. 2(1), 67 (2000)

[15] F.Y. Hsu, M.R. Jolly, J. Campbell, J. Mater. Process. Technol. 209(17), 5736 (2009)

[16] L. Pavlak, Metalurgija 14(1), 31 (2008)

[17] H. Iqbal, A.K. Sheikh, A. Al-Yousef, M. Younas, Mater. Manuf. Process. 27(7), 775 (2012)

[18] C. Reilly, N.R. Green, M.R. Jolly, J.C. Gebelin, Appl. Math. Model. 37(18), 8451 (2013)

[19] W.D. Griffiths, N.W. Lai, Metall. Mater. Trans. A 38(1), 190 (2007)

[20] J. Campbell, M. Tiryakioğlu, Metall. Mater. Trans. B 43(4), 902 (2012)

[21] S.G. Liu, F.Y. Cao, X.Y. Zhao, Y.D. Jia, Z.L. Ning, J.F. Sun, Mater. Sci. Eng. A 626, 159 (2015)

[22] G.E. Bozchaloei, N. Varahram, P. Davami, S.K. Kim, Mater. Sci. Eng. A 548, 99 (2012)

[23] J. Campbell, Castings (Butterworth-Heinemann, Oxford, 2003), p. 31

[24] M. Tiryakioğlu, J. Campbell, Metall. Mater. Trans. A 41(12), 3121 (2010)

[25] H. Hashemi, R. Raiszadeh, J. Appl. Sci. 9(11), 2115 (2009)

[26] A.H. Zadeh, J. Campbell, AFS Trans. 2, 1 (2002)

[27] W. Li, J. Guo, Y. Zhao, H. Zhou, Cfhi Technol. 6, 31 (2013) 